San Miguel, E. [et al.]. Blockchain-enabled participatory incentives for crowdsourced mesh networks. A: International Conference on Economics of Grids, Clouds, Systems and Services. "Economics of Grids, Clouds, Systems, and Services, 16th International Conference, GECON 2019: Leeds, UK, September 17-19, 2019: proceedings". Berlín: Springer, 2019, p. 178-187. ISBN 978-3-030-36027-6

The final authenticated version is available online at https://doi.org/10.1007/978-3-030-36027-6_15.

\title{
Blockchain-Enabled Participatory Incentives for Crowdsourced Mesh Networks
}

\author{
Elena San Miguel ${ }^{1}$, Roxane Timmerman ${ }^{2}$, Sergio \\ Mosquera $^{1}$, Emmanouil Dimogerontakis ${ }^{3}$, Felix Freitag $^{3}$, and Leandro Navarro ${ }^{3}$ \\ 1 Universitat Politecnica de Catalunya \\ \{elena.san.miguel, sergio.mosquera\}@est.fib.upc.edu \\ 2 Ecole Centrale de Marseille \\ roxane.timmerman@ec-m.fr \\ 3 Universitat Politecnica de Catalunya \\ \{edimoger, leandro,felix\}@ac.upc.edu
}

\begin{abstract}
Crowdsourced mesh networks are built, maintained and used by several participants that cooperate to provide and consume connectivity. Providers of infrastructure want to get compensation for their investments and earn tokens; users or consumers want the network to expand for improving the coverage of connectivity and stability. How do we collect funds from consumers and distribute them to providers, guaranteeing satisfaction of every participant? For that, we need of a system that coordinates the flow of economic value in mesh networks in a way that is not only transparent, automated, decentralized and secure, but also beneficial to all. We designed a new economic protocol called Fair to compensate providers for their investments. The key point of our model is that each provider will be paid with different prices for the forwarded traffic: the more devices a provider has, the higher its price/MB forwarded is, up to a certain limit. We implemented the model using MeshDApp, a local blockchain platform for mesh networks. Simulations show how our proposal ensures a win-win situation where the network grows and the providers are compensated for their investment. Also, continuous growth is incentivized while centralization due to few large providers controlling the network is avoided.
\end{abstract}

Keywords: Pricing.Mesh networks·Blockchain.Crowdsourcing

\section{Introduction}

A mesh network has a topology in which each node (router) is capable of relaying data for others. All nodes cooperate in the distribution of data throughout the network for the mutual benefit of its participants. With each participating node, the reach, throughput and resilience of the network expands. With sufficient benefits to participation, a mesh network can quickly grow to provide shared connectivity and at a much cheaper rate than a centralised topology [1]. Figure 1 illustrates the main components of a crowdsourced mesh network. The network infrastructure is composed by several devices (routers) that are owned by different providers, and the consumers connect to the network through any of the providers and use the infrastructure to access any of the services available in the network (e.g. Internet access service in this example). 
Mesh networks have the challenge to scale in absence of economic sustainability. The toughest aspects to deal with is the trust in the agreements between peers and how to ensure the economic sustainability of this collective effort and the balance between contribution and consumption. For instance, as an example scenario and mechanism for economic sustainability, we consider the economic compensation system used in Guifi.net [2]. The aim is to find a balance between total resource contribution and its consumption. Currently, the above described economic compensation system is done manually: each participant declares its costs and consumption (traffic) and then the Guifi.net foundation validates this claim by cross checking it with their own network traffic measurement data and network inventory, according to the agreed list of standard costs. There is, however, room for error or manipulation. The correct application of the compensation system is critical for the economic sustainability of the network, as well as for the return of previous or future investments and maintenance.

Therefore, we agree that there is a need for an automated mechanism where diverse participants, providers and consumers, can pool resources with the confidence that the consumption is i) accounted fairly, without discrimination and proportionally to effort and value, and that ii) these calculations and money transfers are safe, automated, irreversible and shared across different participants, to avoid the cost, delays, errors and potential mistrust from manual accounting and external payments. MeshDApp ${ }^{4}$ is a value transfer platform for mesh networks that uses a local blockchain.

Our work addresses the economic sustainability problem of mesh networks. We designed and implemented a new economic model called Fair using MeshDApp. The goal of our model is to build a win-win situation for the network and the users. First, Section 2 presents the related work. In the Section 3, we present our proposed economic model called Fair and we evaluate the model in Section 4. Then we underline some discussion and future work about our work in Section 5 and conclude in Section 6.

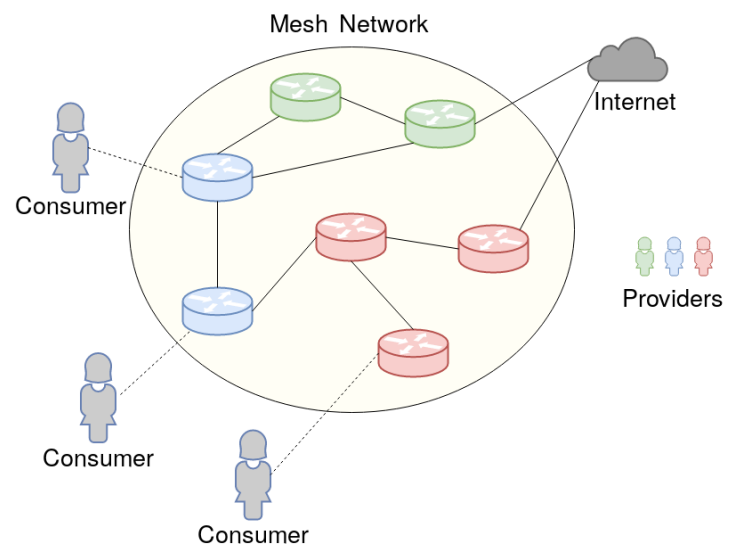

Fig. 1. A mesh network scenario where the infrastructure is owned by different providers.

\footnotetext{
${ }^{4}$ http://dsg.ac.upc.edu/meshdapp
} 


\section{Related Work}

Other projects explore aspects of how mesh networks can be combined with blockchain to provide connectivity under a decentralized economic model involving independent providers and consumers of a crowdsourced network.

Our design relies on a local blockchain infrastructure that operates over an Ethereum-based lightweight consensus protocol (permissioned and low overhead, PoA). A possible alternative to this approach could be Hyperledger Fabric [3]. There are other projects in development that combine the payment in mesh networks with blockchain. Althea Mesh [4] provides last-mile connectivity for the Internet access, where routers pay each other for bandwidth using cryptocurrency payment channels. RightMesh [5] is an ad-hoc mobile mesh networking platform and protocol using the public Ethereum blockchain and RMESH tokens. AmmbrTech [6] has provided initial support for the research of MeshDApp and therefore the results will inspire their evolution.

Several studies provide economic analysis and designs for resource trading. The MeshDApp system is inspired by the experience of our research group in participating in the governance, operations and economic analysis of the Guifi.net community network [2]. The following works applied to related problems have influenced the design of MeshDApp. Route Bazaar [7] is a backward-compatible system for flexible Internet connectivity, which is inspired by the decentralised construction of trust in cryptocurrencies. It looks at agreements among Autonomous Systems with automatic means to form, establish, and verify end-to-end connectivity agreements. Tycoon [8] is a market based distributed resource allocation system based on proportional share with resource auctions for computing or storage. Request Network [9] is a decentralised network that allows anyone to request a payment (a Request Invoice) for which the recipient can pay in a secure way. Request can be seen as a layer on top of Ethereum which allows requests for payments that satisfy a legal framework.

\section{$3 \quad$ Fair Economic Model}

A key goal of our economic model is to promote the expansion of the network coverage awarding providers with a different price per MB forwarded, according to the number of devices they operate. Formalizing the model into a sentence:

The more devices a provider $i$ has, the higher its Price/MB $P_{i}$ is; up to a limit. Figure 2 shows this relation. In fact, we want to build a win-win situation where the growth of the network contributes to more stability and provides more content for users. There are several assumptions in our model:

As it is not good for the network that one provider owns too many devices, we defined a price limit. As soon as the number of devices of a provider is greater than or equal to a given parameter Incentive $(I)$, its Price $/ M B(P)$ is equal to $P_{\max }$. The two goals of this limit are to avoid monopolies (i.e. the network is not dependant on a single provider) and prevent unfair discrimination.

The incentive parameter $I$ is proportional to the ratio of network devices versus providers, so we reward networks with providers with multiple devices, with a constant factor of $3 / 2$ we determined experimentally, defined by Equation 2 . 
To make sure prices cover minimum costs, we also fixed a $P_{\min }$ which is the price per MB forwarded that a provider with a single device would have. We decided to use a linear step function for a number of devices lower than the $I$ parameter, but other shapes, such as an exponential form could be considered in alternative models. Finally, the price per MB forwarded of a provider $i$ is defined by Equation 1 when the number of devices $D_{i} \leq I$ or higher.

$$
P_{i}=\left\{\begin{array}{l}
\left\lfloor P_{\min }+\frac{P_{\max }-P_{\min }}{I-1} \times\left(D_{i}-1\right)\right\rfloor \\
P_{\text {max }}
\end{array}\right.
$$

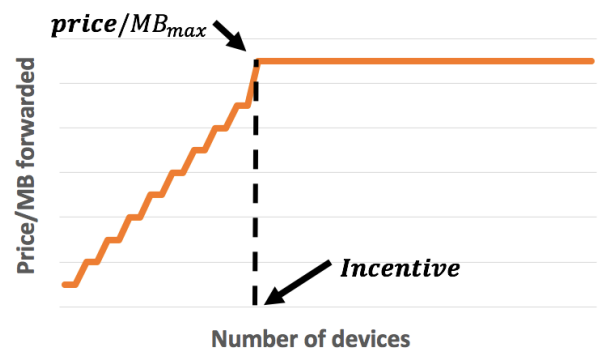

Fig. 2. Price $/ M B$ of a provider under its number of devices.

We do not model the payments from consumers. We simply assume "infinite funds" in a common consumer account, assuming a subscription is paid by clients at each iteration in exchange of clients having unlimited network access. That price varies depending on the total traffic in the network during the last iteration.

\section{Evaluation}

In order to evaluate our economic model we performed simulation. This section presents the design of these experiments and their results.

\subsection{Design of experiments}

The simulation is a test constructed as the sequential execution of the activities that take place during a day, repeated during one month. The first activity consists on getting the monitored traffic values for all devices. Then, the prices per MB per provider are computed and the charges (owed tokens) are calculated for all providers. After that, the payment to the providers is carried out taking advantage of the amount deposited in a common funds account. Finally, the providers pay the costs of maintenance of their devices and buy new devices.

We have decided to perform one iteration per day (the data is pre-processed so that we have a given amount of $\mathrm{MB}$ forwarded per provider, for each day). The two following subsections present the inputs of these simulation and the measurements we performed. 


\subsection{Inputs}

Economic Models: We performed our simulation for the Fair economic model, and two variants, one with unlimited $P_{\max }$ (unbounded), and another with the same price for all providers (fixed-price).

Data: We used real data from the QMPSU mesh network in Barcelona. We had received $(r x)$ and transmitted $(t x)$ bytes for 62 devices, every hour during one month. We assumed that forwarded data $(f x)$ is calculated as $\min (r x, t x)$. Although this solution is not entirely realistic, we assume is fair enough to have values related to real traffic.

Variables: Price of a new device: We fixed the price of a new device to a proportionally value of $10^{9}$ tokens. We assumed that a monthly cost for a provider is around $4 €$, and the price of a new device is $150 €$. Then, we calculate the cost of any provider $i$ with the formula $\operatorname{Cost}_{i}=0.9 *\left(f x * P_{\text {min }}\right)$. Indeed, it is essential for a provider who owns a single device to be able to get a benefit (TotalReceived-Cost). Otherwise, he will not be able to invest in the network due to lack of money. Size of the network: We performed the simulation with 2, 5 or 10 providers for each variant of the economic model.

Initial state: In the first iteration, half of the providers start with 1 device, and half start with 3 devices. This choice enables the system to have different providers at the beginning, in terms of importance in the network, to observe their developments and to discover whether it has an impact or not.

Model of providers' behaviors: One of Fair's goals is to incentivize providers, so that they can invest in the network buying new devices. In order to evaluate the efficiency of the way this economic model incentivizes them, we have to model providers' behaviors: do they buy new devices? When? We decided to model it as follows. A provider $i$ buys a new device when he has enough tokens $\left(\sharp\right.$ Tokens $\left._{i} \geq 10^{9}\right)$, with a probability $p 1=0.7$ or $p 2=0.2$ depending on the number of Devices . $_{\text {. }}$

In fact, if the number of devices $D_{i} \leq I, P_{i}$ will increase if the provider $i$ decides to buy a new device and so, he is incentivized to do it. We modelled it with the probability $p 1$. Otherwise, his $P_{i}$ will be constant and would have less reasons to buy a new device. Therefore, this happens with probability $p 2 \leq p 1$.

In one hand, the Unbounded model, a provider is always incentivized to buy a new device. Therefore, the probability is $p=p 1=0.7$. On the other hand, in the Fixed-Price model the incentive disappears. Therefore, the probability is $p=p 2=0.2$.

\subsection{Metrics}

We evaluated the Fair economic model looking at 3 metrics that change over time: the size of the network, the number of tokens per provider, and the number of devices per provider.

The size of the network over time is a metric that allows us to evaluate if an economic model incentivizes efficiently providers to buy new devices and help the network grow. This is a part of the evaluation of the win-win situation desired. In fact, the bigger the network is, the bigger the stability it offers to its consumers.

The number of tokens per provider over time is a measurement that lets us to evaluate the second part of the win-win situation. In fact, a provider investing in the network should be rewarded earning tokens. 
Finally, the number of devices per provider over time is a measurement that makes it possible to evaluate whether the limit $P_{\max }$ is efficient or not. In fact, it should avoid monopoly and therefore, dependence of the network on a single provider.

\subsection{Results}

Figure 3 shows how Fair incentivizes the providers to invest in the network. In fact, the growth is faster than with a Fixed-Price model (we can observe how the deviation between the two curves increases). The growth is even faster with the Unbounded model, as expected. The drawbacks of this last model will be discussed later.

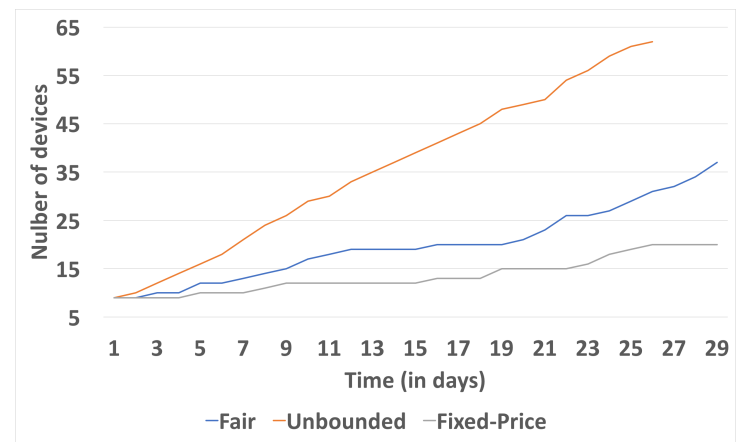

Fig. 3. Total number of devices in a network of 10 providers over time.

First of all, Figure 4 shows how the limit Incentive is efficient. In fact, it seems that the maximum number of devices $D_{\max }$ tends to get deeply below the incentive $I$ line throughout the time. It shows also why our economic model is fair. For instance, Provider 3 has a single device at the first iteration and has $D_{\max }=11$ devices at the end of the simulation. It proves that Fair places no one at a disadvantage. Then, Figure 5 shows the biggest drawback of the Unbounded model: the creation of a monopoly and the difficulty of little providers to grow (Providers 1 and 5 have 2 and 1 devices at the end of the simulation, respectively). It remarks the importance of the $I$ threshold in Fair, which avoids the creation of monopolies and the acceleration of differences of importance between providers. Figure 6 shows how providers slowly invest in the network in absence of an incentive.

Finally, Figures 7 and 8 shows that observations made in Figure 6 are confirmed by our experiment with other network sizes.

Figure 9 shows how Fair fulfils all conditions to ensure a win-win situation since it also makes possible that provider earns tokens faster than with the Fixed-Price model.

As a conclusion, we can state that the results of the simulation show how Fair ensures a win-win situation where providers invest in the network and earn tokens, avoiding monopoly at the same time. 


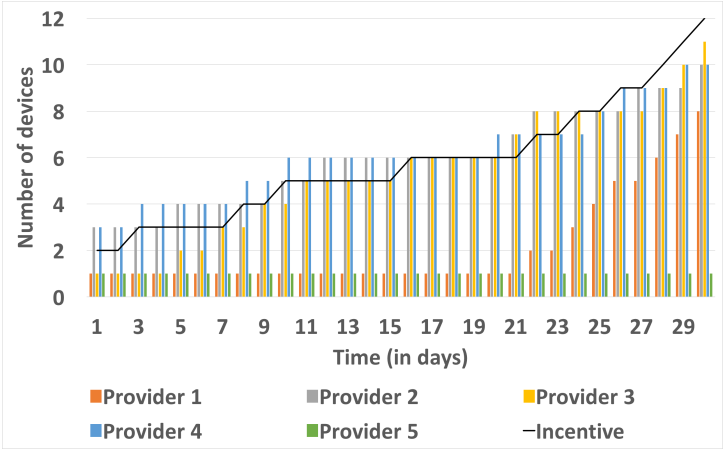

Fig. 4. Number of devices per provider over time with the Fair Model

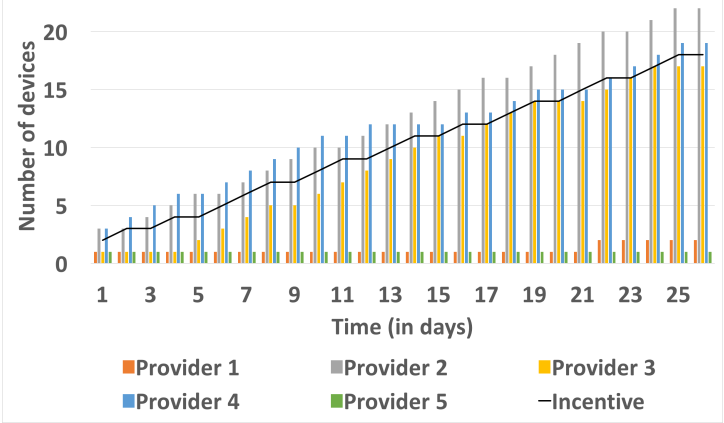

Fig. 5. Number of devices per provider over time with the Unbounded Model

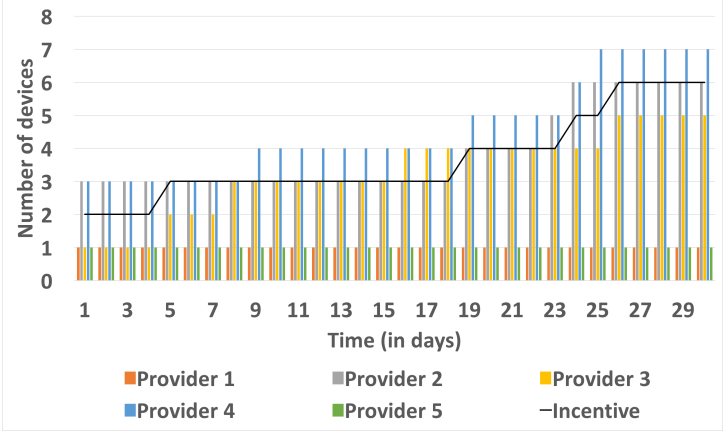

Fig. 6. Number of devices per provider over time with the Fixed-price Model

\section{Discussion and Future Work}

There are still a lot of work that could be developed in order to test and validate the solution we proposed. 


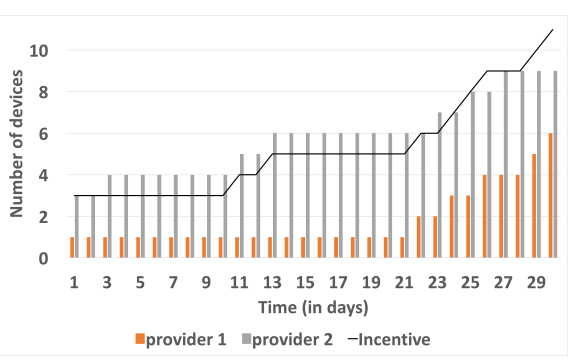

Fig. 7. Number of devices per provider over time with the Fair model in a network of 2 providers.

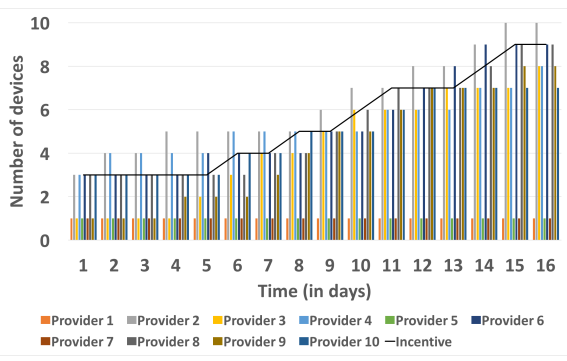

Fig. 8. Number of devices per provider over time with the Fair model in a network of 10 providers.

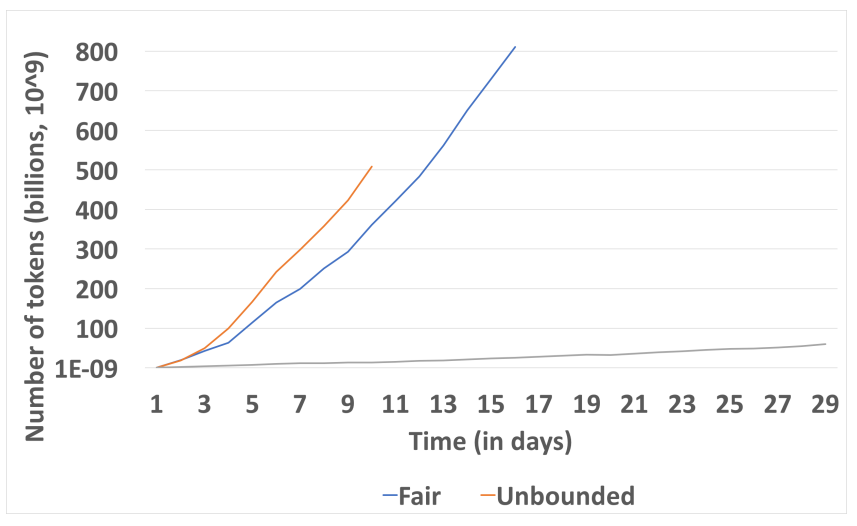

Fig. 9. Mean number of tokens per provider in a network of 10 providers over time.

First of all, with the same simulation, it is crucial to design and adopt a more realistic probability distribution in order to model providers' behaviors. In fact, it would help us to evaluate the incentives in the model in a more efficient way. It would also be interesting to test it in a real environment for the same reason, and also for being able to work with real data. For instance, for the results presented in the current paper, it may be noted that provider 3 always buys a lot of devices. This could be caused by the data we are using, as it may give a significantly high number of $\mathrm{MB}$ forwarded for this provider. Using real data would allow us to derive more confident conclusions about the efficiency and the "fairness" of Fair.

Then, there are several parameters in Section 3 that could be analyzed, tested and optimized. For example, it would be interesting to test the use of a quadratic function or to change the definition of the $I$ incentive. Moreover, the definition we give for fairness (incentivize providers to promote the network growth and to prevent monopoly and disadvantages for anyone within the network) could be improved and extended.

Finally, other economic models can be envisaged, because during the development of the experiments we had some ideas about alternatives or extensions. For example, we though about a crowd-funding system where each provider, if he wants to, gives 
a part of what it should be paid to the network. Then, this amount of money would be given to a new provider when it joins the network, as a "welcome gift". Donation from providers could also be designed as a tax, that every provider has to pay. All these possibilities are potential future work because they can be merged into the Fair economic model to build a more complete economic model.

\section{Conclusion}

In order to address the need of a system which coordinates the economic value flow of mesh networks in a transparent, automated, decentralized and secure way, we designed and implemented a new economic model called Fair using MeshDApp.

The goal of the model, presented in Section 3, is to create a win-win situation for the network and the users. This aim is enforced by Fair because the main idea is "The more devices a provider has, the higher its price per MB forwarded is; up to a certain limit". This incentivizes providers to invest in the network buying new devices and avoids monopoly and inequalities across providers.

The simulation results show that Fair makes this win-win situation possible. However, in order to validate completely our proposed solution, protocol design improvements, alternatives and experiments have to be completed as future work.

\section{References}

1. Panagiota Micholia, Merkourios Karaliopoulos, Iordanis Koutsopoulos, Leandro Navarro, R Baig, Dimitris Boucas, Maria Michalis, and Panayotis Antoniadis. Community networks and sustainability: a survey of perceptions, practices, and proposed solutions. IEEE Communications Surveys \& Tutorials, PP, 072017.

2. Roger Baig, Lluís Dalmau, Ramon Roca, Leandro Navarro, Felix Freitag, and Arjuna Sathiaseelan. Making community networks economically sustainable, the guifi.net experience. In Proceedings of the 2016 Workshop on Global Access to the Internet for All, GAIA '16, pages 31-36, New York, NY, USA, 2016. ACM.

3. Mennan Selimi, Aniruddh Rao, Anwaar Ali, Leandro Navarro, and Arjuna Sathiaseelan. Towards blockchain-enabled wireless mesh networks. 042018.

4. Althea Mesh. Althea: An incentivized mesh network protocol. [Online]. https://altheamesh.com/documents/whitepaper.pdf.

5. Right Mesh. Rightmesh. [Online]. https://www.rightmesh.io/docs/RightMesh_TW P5.pdf.

6. AmmbrTech SRL. Ammbr network. [Online]. https://ammbrtech.com/.

7. Ignacio Castro, Aurojit Panda, Barath Raghavan, Scott Shenker, and Sergey Gorinsky. Route bazaar: Automatic interdomain contract negotiation. In 15th Workshop on Hot Topics in Operating Systems (HotOS XV), Kartause Ittingen, Switzerland, 2015. USENIX Association.

8. Kevin Lai, Lars Rasmusson, Eytan Adar, Stephen Sorkin, Li Zhang, and Bernardo Huberman. Tycoon: an implementation of a distributed, market-based resource allocation system. Multiagent and Grid Systems, 1, 122004.

9. Request Network. A decentralized network for payment requests. [Online]. https://request.network/assets/pdf/request_whitepaper.pdf. 